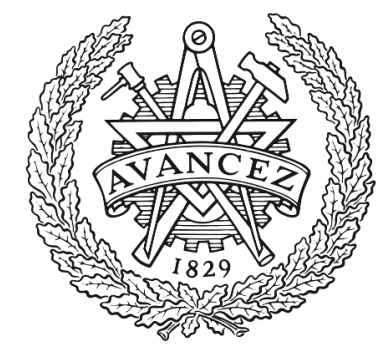

CHALMERS

UNIVERSITY OF TECHNOLOGY

\title{
Cooperative Localization of Vehicles without Inter-vehicle Measurements
}

Downloaded from: https://research.chalmers.se, 2023-04-26 09:34 UTC

Citation for the original published paper (version of record):

Fröhle, M., LINDBERG, C., Wymeersch, H. (2018). Cooperative Localization of Vehicles without Inter-vehicle Measurements. IEEE Wireless Communications and Networking Conference, WCNC. http://dx.doi.org/10.1109/WCNC.2018.8377047

N.B. When citing this work, cite the original published paper. 


\title{
Cooperative Localization of Vehicles without Inter-vehicle Measurements
}

\author{
Markus Fröhle, Christopher Lindberg and Henk Wymeersch \\ Department of Electrical Engineering, Chalmers University of Technology, Gothenburg, Sweden \\ E-mail:\{frohle, chrlin, henkw\}@ chalmers.se
}

\begin{abstract}
While cooperation among vehicles can improve localization, standard communication technologies (e.g., 802.11p) cannot provide reliable range or angle measurements. To allow cooperation without explicit inter-vehicle measurements, we propose a cooperative localization method whereby vehicles track mobile features in the environment and use associations of features among vehicles to improve the vehicles' localization accuracy. The proposed algorithm, which scales linearly in the number of vehicles and quadratically in the number of tracked features, shows superior localization performance compared to a non-cooperative approach.
\end{abstract}

\section{INTRODUCTION}

Intelligent transportation systems (ITS) in general and autonomous driving in particular require accurate position information [1]. On-board sensors, such as a global navigation satellite system (GNSS) receiver, provide absolute position, whereas a radar-like sensor provides relative measurements with respect to moving objects (e.g., pedestrians), termed features, which are not part of any offline map. Such a sensor is capable of delivering high-quality observations of the environment, but suffers from an unknown association between the objects and the measurements, thus requiring a data association step for tracking. When vehicles are equipped with an IEEE $802.11 \mathrm{p}$ radio interface, allowing them to communicate with a road side unit (RSU), i.e., through vehicleto-infrastructure (V2I) communication, or cooperating vehicles in range ( $\mathrm{V} 2 \mathrm{~V}$ communication), they can share measurements. In such a cooperative setting, local measurement associations within a vehicle as well as associations among vehicles must be resolved. Under the assumption that such associations are possible, [2] presented a distributed method for accurate feature and vehicle localization through nonparametric belief propagation (BP) on a factor graph. Moreover, a distributed implementation was provided using a consensus scheme [3]. An adaption for parametric BP was presented and compared to a centralized Kalman filter in [4]. In [5], [6], a centralized nonparametric multi-target tracker (MTT), to track an unknown number of features based on measurements provided by multiple sensors with known location, is presented. Data association (DA) is incorporated in a factor graph (FG) and resolved by BP. Through a redundant formulation of the DA uncertainty, following [7], complexity is kept low and scales quadratically with number of features. This type of filter belongs to the class of joint probabilistic data association (JPDA) filter and the related multiple hypothesis density tracker (MHT) [8]. More recent approaches for MTT are based on finite set statistics (FISST) which involve the probability hypothesis density (PHD) filters and the multi-Bernoulli filters [9], [10], which avoid the DA problem. However, most of these methods are restricted to a single sensor with known location.

In this paper, we follow a cooperative localization approach, where vehicles use their on-board sensors, i.e., GNSS and radar-like sensors, to track the vehicle state as well as features in the environment. All measurements are sent to the RSU using V2I communication. At the RSU, the proposed tracker is executed centrally allowing to improve the localization performance. Our primary objective is not to track the features, but use them to improve the tracking of the vehicle states. In doing so, we remove the assumption of perfect DA from [2], [4]. In contrast to [5], [6], we consider the sensor (vehicle) state to be unknown and time-varying. We apply a nonparametric tracking approach allowing nonlinear vehicle and feature motion and observation models, and resolve DA similarly to [5], [6]. In contrast to other cooperative vehicle localization methods, we do not rely on inter-vehicle measurements (e.g., received signal strength in [11]).

\section{A. Notation}

Boldface lowercase letters $f$ denote column vectors. When applicable to a certain vector quantity, subscript $k$ indicates a reference to feature $k$, subscript $s$ indicates that the vector corresponds to vehicle $s$, subscript $m$ signifies V2F measurement $m$, and $t$ indicates the time instant. For a quantity denoted by $\boldsymbol{f}_{k, s, t}$, this means that it corresponds to feature $k$, vehicle $s$, at time $t$. If one subscript is removed, say $k$ in $f_{s, t}$, this denotes the stacking into a column vector over the $k$ 's, i.e., $\boldsymbol{f}_{s, t}=\left[\boldsymbol{f}_{1, s, t}^{\top}, \ldots, \boldsymbol{f}_{K, s, t}^{\top}\right]^{\top}$. Removing another index produces an additional stacking over that index, e.g., $\boldsymbol{f}_{t}=\left[\boldsymbol{f}_{1, t}^{\top}, \ldots, \boldsymbol{f}_{S, t}^{\top}\right]^{\top}$. For the time index $t$, a stacking over $1, \ldots, t$ is denoted by $\boldsymbol{f}_{1: t}=\left[\boldsymbol{f}_{1}^{\top}, \ldots, \boldsymbol{f}_{t}^{\top}\right]^{\top}$. In Section IV, we shorten the notation by removing the dependence on the state variable of the BP messages and factors, and indicate it by subscript $k$ instead of $\boldsymbol{f}_{k}$, subscript $s$ instead of $\boldsymbol{x}_{s}$, and so on. For example, we substitute $\alpha\left(\boldsymbol{f}_{n, k}\right)$ by $\alpha_{k}$. We also introduce a superscript to indicate iteration index when applicable, for example $\tilde{\alpha}_{k, s}^{(i)}$.

\section{SYSTEM MODEL}

We consider an urban ITS scenario consisting of $S$ vehicles (illustrated in Fig. 1). Each vehicle is equipped with a sensor allowing them to determine their absolute position, (e.g., a 


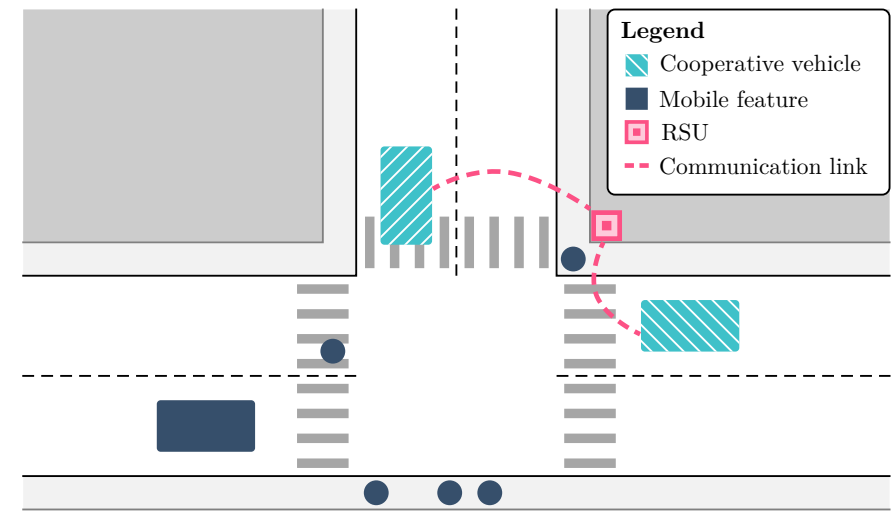

Figure 1. Urban ITS scenario with two vehicles cooperating through the RSU and six mobile features.

GNSS receiver), and a sensor to retrieve relative positions to $K$ features present in the environment (e.g., a radar), through vehicle-to-feature (V2F) measurements. For simplicity of the exposition, we assume that all $S$ vehicles are able to observe the same $K$ features. At every time-step $t$, each vehicle receives a GNSS and V2F measurements with respect to all $K$ features. The time-varying state $\boldsymbol{x}_{s, t} \in \mathbb{R}^{N}$ of vehicle $s$ at time instant $t$ is statistically modeled as $p\left(\boldsymbol{x}_{s, t} \mid \boldsymbol{x}_{s, t-1}\right)$, and similarly for each feature state $k$ as $p\left(\boldsymbol{f}_{k, t} \mid \boldsymbol{f}_{k, t-1}\right)$. We define the joint state of the whole system at time $t$ to be $\boldsymbol{\theta}_{t} \triangleq\left[\boldsymbol{x}_{t}^{\top}, \boldsymbol{f}_{t}^{\top}\right]^{\top}$. The GNSS measurement of vehicle $s$ at time $t$ is modeled with the likelihood function $p\left(\boldsymbol{z}_{s, t}^{(\mathrm{G})} \mid \boldsymbol{x}_{s, t}\right)$, where $\boldsymbol{z}_{s, t}^{(\mathrm{G})}$ denotes the GNSS measurement. The $m$-th V2F measurement between vehicle $s$ and feature $k$ at time $t$ is modeled as

$$
\boldsymbol{z}_{m, s, t}^{(\mathrm{V} 2 \mathrm{~F})}=q_{k, s}\left(\boldsymbol{f}_{k, t}, \boldsymbol{x}_{s, t}\right)+\boldsymbol{w}_{k, s, t}^{(\mathrm{V} 2 \mathrm{~F})}
$$

where $q_{k, s}(\cdot, \cdot)$ is a possibly nonlinear function and $\boldsymbol{w}_{k, s, t}^{(\mathrm{V} 2 \mathrm{~F})}$ represents the $\mathrm{V} 2 \mathrm{~F}$ measurement noise which is independent and identically distributed (i.i.d.) over time and space. Note that $m$ is used for $\mathrm{V} 2 \mathrm{~F}$ measurements since the order of $\boldsymbol{z}_{s, t}^{(\mathrm{V} 2 \mathrm{~F})}$ may not coincide with the order in $\boldsymbol{f}_{t}$, i.e., measurements may arrive randomly permuted in each time-step due the radarlike sensor, and feature and vehicle mobility. We define the combined measurement vector $\boldsymbol{z}_{m, s, t}=\left[\boldsymbol{z}_{s, t}^{(\mathrm{G}), \mathrm{T}}, \boldsymbol{z}_{m, s, t}^{(\mathrm{V} 2 \mathrm{~F}), \mathrm{T}}\right]^{\mathrm{\top}}$. Furthermore, we assume that all measurements (GNSS and V2F) from all vehicles are communicated to the RSU instantaneously and without errors.

\section{PROBlem Formulation IN FACTOR GRAPH FORM}

In this section, we describe the cooperative localization problem subject to the system model of Section II. We then utilize the structure of the problem to factorize the joint posterior PDF, which is graphically visualized by a factor graph.

\section{A. Optimal State Estimation}

The state estimation we seek is the minimum mean-square error (MMSE) estimator $\hat{\boldsymbol{\theta}}_{t}^{\text {(MMSE) }}$ of $\boldsymbol{\theta}_{t}$ containing the states of the vehicles and features, which in a Bayesian framework is given by $\hat{\boldsymbol{\theta}}_{t}^{(\mathrm{MMSE})} \triangleq \int \boldsymbol{\theta}_{t} p\left(\boldsymbol{\theta}_{t} \mid \boldsymbol{z}_{1: t}\right) \mathrm{d} \boldsymbol{\theta}_{t}$. Hence, this task includes the calculation of the posterior distribution $p\left(\boldsymbol{\theta}_{t} \mid \boldsymbol{z}_{1: t}\right)$ of the joint state given all measurements up to time $t$. We will first determine a factorization of the joint probability density function (PDF) of $p\left(\boldsymbol{\theta}_{1: t}, \boldsymbol{a}_{1: t}, \boldsymbol{b}_{1: t} \mid \boldsymbol{z}_{1: t}\right)$, where $\boldsymbol{a}_{t}$ and $\boldsymbol{b}_{t}$ are association variables (to be defined), after which $p\left(\boldsymbol{\theta}_{t} \mid \boldsymbol{z}_{1: t}\right)$ is found through marginalization.

Since a feature can generate only one measurement at each vehicle, and a measurement at a vehicle can only originate from one feature [12], we define the association variables following the approach of [6], [7]: $a_{k, s, t} \triangleq m \in\{1, \ldots, K\}$ and $b_{m, s, t} \triangleq k \in\{1, \ldots, K\}$. The value of $a_{k, s, t}$ indicates which measurement $m$ feature $k$ generates at vehicle $s$ at time $t$, while the value of $b_{m, s, t}$ indicates which feature $k$ generates measurement $m$ at vehicle $s$ at time $t$. Note that the above formulation gives a one-to-one mapping between $a_{k, s, t}$ and $b_{m, s, t}$, such that if $a_{k, s, t}$ is known, so is $b_{m, s, t}$.

\section{B. A Priori Distribution}

Given the structure of the problem, the a priori PDF of the joint state of the whole system factorizes as

$$
\begin{aligned}
p\left(\boldsymbol{\theta}_{1: t}\right) & =p\left(\boldsymbol{x}_{1: t}\right) p\left(\boldsymbol{f}_{1: t}\right) \\
& =\left(\prod_{s=1}^{S} p\left(\boldsymbol{x}_{s, 0}\right) \prod_{t^{\prime}=1}^{t} p\left(\boldsymbol{x}_{s, t^{\prime}} \mid \boldsymbol{x}_{s, t^{\prime}-1}\right)\right) \\
& \times\left(\prod_{k=1}^{K} p\left(\boldsymbol{f}_{k, 0}\right) \prod_{t^{\prime}=1}^{t} p\left(\boldsymbol{f}_{k, t^{\prime}} \mid \boldsymbol{f}_{k, t^{\prime}-1}\right)\right),
\end{aligned}
$$

where $p\left(\boldsymbol{x}_{s, 0}\right)$ is the prior PDF of the state of vehicle $s$, and $p\left(\boldsymbol{f}_{k, 0}\right)$ the prior PDF of the state of feature $k .{ }^{1}$ For the association variables, their definition implies

$$
p\left(\boldsymbol{a}_{1: t}, \boldsymbol{b}_{1: t}\right)=\prod_{t^{\prime}=1}^{t} \prod_{s=1}^{S} \psi\left(\boldsymbol{a}_{s, t^{\prime}}, \boldsymbol{b}_{s, t^{\prime}}\right),
$$

with

$$
\psi\left(\boldsymbol{a}_{s, t}, \boldsymbol{b}_{s, t}\right)=\prod_{k=1}^{K} \prod_{m=1}^{M} \Psi\left(a_{k, s, t}, b_{m, s, t}\right),
$$

wherein

$$
\Psi\left(a_{k, s, t}, b_{m, s, t}\right)=\left\{\begin{array}{cc}
0, & a_{k, s, t}=m, b_{m, s, t} \neq k \\
\quad \text { or } a_{k, s, t} \neq m, b_{m, s, t}=k \\
1, \quad \text { otherwise. }
\end{array}\right.
$$

\section{Measurement Likelihood}

Conditioned on the association variable $\boldsymbol{a}$, the measurement likelihood of the $\mathrm{V} 2 \mathrm{~F}$ measurements from the initial time up to time $t$ is given by

$$
\begin{aligned}
& p\left(\boldsymbol{z}_{1: t}^{(\mathrm{V} 2 \mathrm{~F})} \mid \boldsymbol{f}_{1: t}, \boldsymbol{x}_{1: t}, \boldsymbol{a}_{1: t}\right)= \\
& \prod_{t^{\prime}=1}^{t} \prod_{s=1}^{S} \prod_{k=1}^{K} p\left(\boldsymbol{z}_{a_{k, s, t^{\prime}}, s, t^{\prime}}^{(\mathrm{V} 2 \mathrm{~F})} \mid \boldsymbol{f}_{k, t^{\prime}}, \boldsymbol{x}_{s, t^{\prime}}\right),
\end{aligned}
$$

\footnotetext{
${ }^{1}$ In the absence of prior information, the vehicle prior may be found by initializing the vehicle state by the GNSS measurement with a large enough support to cover the region of interest. The feature prior may be found by initializing the feature state by the GNSS plus V2F measurement, also with a large enough support.
} 
while for the GNSS measurements, we find that

$$
p\left(\boldsymbol{z}_{1: t}^{(\mathrm{G})} \mid \boldsymbol{x}_{1: t}\right)=\prod_{t^{\prime}=1}^{t} \prod_{s=1}^{S} p\left(\boldsymbol{z}_{s, t^{\prime}}^{(\mathrm{G})} \mid \boldsymbol{x}_{s, t^{\prime}}\right) .
$$

Note that GNSS measurements are completely resolved, since they observe a single known target, i.e., there is no DA uncertainty.

\section{Joint Posterior and Factor Graph}

Due to the Markovian and independence assumptions, the complete factorization of the posterior PDF for vehicle and feature states, as well as the DA, is given by

$$
\begin{aligned}
& p\left(\boldsymbol{\theta}_{1: t}, \boldsymbol{a}_{1: t}, \boldsymbol{b}_{1: t} \mid \boldsymbol{z}_{1: t}\right) \propto\left(\prod_{k=1}^{K} p\left(\boldsymbol{f}_{k, 0}\right) \prod_{t^{\prime}=1}^{t} p\left(\boldsymbol{f}_{k, t^{\prime}} \mid \boldsymbol{f}_{k, t^{\prime}-1}\right)\right. \\
& \left.\times \prod_{s=1}^{S} p\left(\boldsymbol{z}_{a_{k, s, t^{\prime}}, s, t^{\prime}}^{(\mathrm{V} 2 \mathrm{~F})} \mid \boldsymbol{f}_{k, t^{\prime}}, \boldsymbol{x}_{s, t^{\prime}}\right) \prod_{m=1}^{K} \Psi\left(a_{k, s, t^{\prime}}, b_{m, s, t^{\prime}}\right)\right) \\
& \times \prod_{s=1}^{S} p\left(\boldsymbol{x}_{s, 0}\right) \prod_{t^{\prime}=1}^{t} p\left(\boldsymbol{x}_{s, t^{\prime}} \mid \boldsymbol{x}_{s, t^{\prime}-1}\right) p\left(\boldsymbol{z}_{s, t^{\prime}}^{(\mathrm{G})} \mid \boldsymbol{x}_{s, t^{\prime}}\right) .
\end{aligned}
$$

Note that in the likelihoods, we only need to condition on one of the data association variables, $\boldsymbol{a}_{1: t}$ or $\boldsymbol{b}_{1: t}$, since $\boldsymbol{b}_{1: t}$ can be recovered from knowing $a_{1: t}$, and vice versa. The reason for introducing both $\boldsymbol{a}_{t}$ and $\boldsymbol{b}_{t}$ is that it allows for a factorization in (9) using the previously described factors $\Psi(\cdot, \cdot)$, with which message-passing can efficiently approximate the associations. In contrast, brute force exact calculation of the data association becomes intractable for all but the smallest problems [7]. Note also that the feature-measurement data association variable $\boldsymbol{a}$ is independent over time, since the order in which V2F measurements are retrieved is i.i.d. over time.

In Fig. 2, the factor graph of this factorization is illustrated. In the next section, we describe how to estimate the marginal PDFs that we seek, in a computationally efficient way, through message passing on the factor graph. We have introduced notational shorthands $v_{k}$ for $p\left(\boldsymbol{z}_{a_{k, s, t}, s, t}^{(\mathrm{V} 2 \mathrm{~F})} \mid \boldsymbol{f}_{k, t}, \boldsymbol{x}_{s, t}\right)$ and $u_{s}$ for $p\left(\boldsymbol{z}_{s, t}^{(\mathrm{G})} \mid \boldsymbol{x}_{s, t}\right)$.

\section{NonParametric BP Message Passing}

In this section, we detail the computation steps to calculate the marginals of (9), required for the MMSE estimator from Section III-A using loopy BP. For tree-like FGs, BP can be used to efficiently compute exact marginal distributions. However, the FG described by the factorization (9) contains cycles (between factors $\boldsymbol{a}$ and $\boldsymbol{b}$, as well as among sensors) and we can apply BP by ignoring the presence of cycles in the FG. This is known as loopy BP, where its solution yields a belief, not necessarily the true marginal distribution. However, in practice the procedure often arrives at a reasonable set of approximations of the correct marginals [13]. For details regarding loopy BP, see for example [13].

\section{A. Multisensor Localization through Multitarget Tracking}

We divide the proposed algorithm into six steps, each consisting of a number of message updates. Messages are only updated forward in time, equivalent to a filtering approach [14]. Messages are shown in shorthand in Fig. 2.

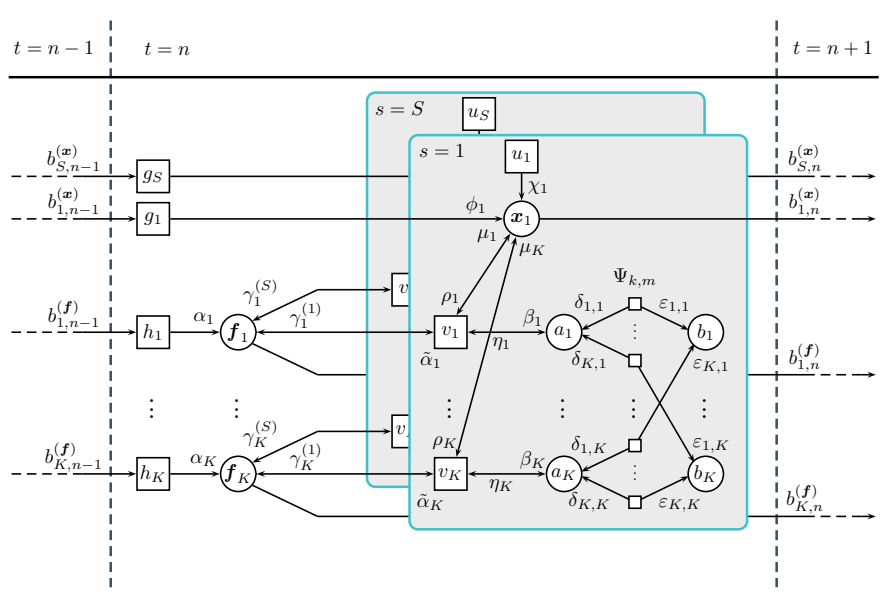

Figure 2. Factor graph representation of the posterior PDF (9). A label close to a vertex represents an incoming message to that vertex. Note that messages are functions of the variable appearing in the adjacent variable vertex. In the figure, we let $h_{k}=p\left(\boldsymbol{f}_{k, t} \mid \boldsymbol{f}_{k, t-1}\right)$, and $g_{s}=p\left(\boldsymbol{x}_{s, t} \mid \boldsymbol{x}_{s, t-1}\right)$.

Step 1 - Prediction: This step involves computing $\alpha_{k, t}\left(\boldsymbol{f}_{k, t}\right)$ as the message representing the prediction of feature $k$ to time instant $t$ from the belief $b_{k, t-1}^{(\boldsymbol{f})}\left(\boldsymbol{f}_{k, t-1}\right)$ with the help of the state-space model. It is computed as

$$
\alpha_{k, t}\left(\boldsymbol{f}_{k, t}\right)=\int p\left(\boldsymbol{f}_{k, t} \mid \boldsymbol{f}_{k, t-1}\right) b_{k, t-1}^{(\boldsymbol{f})}\left(\boldsymbol{f}_{k, t-1}\right) \mathrm{d} \boldsymbol{f}_{k, t-1} .
$$

In a similar manner, we have a parallel prediction step of the vehicle state, where we compute $\phi_{s, t}\left(\boldsymbol{x}_{s, t}\right)$ as

$$
\phi_{s, t}\left(\boldsymbol{x}_{s, t}\right)=\int p\left(\boldsymbol{x}_{s, t} \mid \boldsymbol{x}_{s, t-1}\right) b_{s, t-1}^{(\boldsymbol{x})}\left(\boldsymbol{x}_{s, t-1}\right) \mathrm{d} \boldsymbol{x}_{s, t-1} .
$$

Note that $b_{k, 0}^{(\boldsymbol{f})}\left(\boldsymbol{f}_{k, 0}\right)=p\left(\boldsymbol{f}_{k, 0}\right)$ and $b_{s, 0}^{(\boldsymbol{x})}\left(\boldsymbol{x}_{s, 0}\right)=p\left(\boldsymbol{x}_{s, 0}\right)$, and we assume that the RSU has access to the relevant priors. From now on we drop the time index $t$, as we stay in this time slot for the rest of the algorithm description.

Step 2 -Measurement evaluation: Next, we compute the outgoing message from $\boldsymbol{f}_{k}$ to $v_{k, s}$ which is $\tilde{\alpha}_{k, s}$. With the fact that $\gamma_{k, s}^{(0)}=1$, we have

$$
\tilde{\alpha}_{k, s}^{(0)}=\alpha_{k} \prod_{s^{\prime} \neq s} \gamma_{k, s^{\prime}}^{(0)}=\alpha_{k}
$$

Furthermore, to compute $\rho_{k, s}^{(1)}$ we use message $\chi_{s}$ and initialize all $\mu_{k, s}$ as $\mu_{k, s}^{(0)}=1$, which yields

$$
\rho_{k, s}^{(0)}=\phi_{s} \chi_{s} \prod_{k^{\prime} \neq k} \mu_{k^{\prime}, s}=\phi_{s} \chi_{s} .
$$

Now, using (12), and (13) to compute $\beta_{k, s}$ we have

$$
\beta_{k, s}=\iint v_{k} \phi_{s} \chi_{s} \alpha_{k} \mathrm{~d} \boldsymbol{f}_{k} \mathrm{~d} \boldsymbol{x}_{s} .
$$

The message $\beta_{k, s}$ carries information about data association given the observed measurements and the predicted and estimated states. 
Step 3 - Initialization of DA: The data association message passing follows the approach described in [5]-[7], with an initialization step, followed by an iterative loop of alternating between updating messages $\delta_{m, k, s}$ and $\varepsilon_{k, m, s}$. The DA loop is performed per sensor, therefore, we drop the subscript $s$ on the messages $\delta_{m, k, s}$ and $\varepsilon_{m, k, s}$. We also introduce the auxiliary backward-messages $\tilde{\delta}_{m, k}$, which go in the opposite direction to $\delta_{m, k}$. The DA procedure is initialized from $\varepsilon_{k, m}^{(0)}$ as

$$
\varepsilon_{k, m}^{(0)}=\sum_{a_{k, s}} \Psi_{k, m} \tilde{\delta}_{m, k},
$$

in which (using here that the $\delta_{m, k}$ are initialized as 1)

$$
\tilde{\delta}_{m, k}=\beta_{k, s} \prod_{m^{\prime} \neq m} \delta_{m^{\prime}, k}=\beta_{k, s} .
$$

We plug this into (15) to obtain

$$
\varepsilon_{k, m}^{(0)}=\sum_{a_{k}} \Psi_{k, m} \beta_{k, s} .
$$

Step 4 - DA iterative update loop: Once initialization of the DA loop is performed, we update $\delta_{m, k}^{(p)}$ iteratively, for iterations $p=1, \ldots, P$, according to

$$
\delta_{m, k}^{(p)}=\sum_{b_{m}} \Psi_{k, m} \prod_{k^{\prime} \neq k} \varepsilon_{k^{\prime}, m}^{(p-1)} .
$$

With the expression of $\delta_{m, k}^{(p)}$ we can now update our calculation of $\varepsilon_{k, m}^{(p)}$ by

$$
\varepsilon_{k, m}^{(p)}=\sum_{a_{k}} \Psi_{k, m} \beta_{k, s} \prod_{m^{\prime} \neq m} \delta_{m^{\prime}, k}^{(p)} .
$$

After $P$ iterations, we complete the data association loop and compute the outgoing message $\eta_{k, s}$ as (reintroducing the $s$ subscript on the DA messages)

$$
\eta_{k, s}=\prod_{m=1}^{K} \delta_{m, k, s}^{(P)} .
$$

An interpretation of $\eta_{k, s}$ is that it contains the approximate data association information after applying the MTT exclusion assumption, using the information from $\beta_{k, s}$. An efficient implementation of the DA procedure is stated in [15].

Step 5 - Measurement correction loop using DA: Now, we take the information we have about the DA in the form of $\eta_{k, s}$ and compute the outgoing message $\gamma_{k, s}$, which is to say we update our measurement likelihood with new DA information. Then, we take an iterative approach to updating the messages in the network carrying information between vehicle and feature states. The updating steps in the loop are summarized as follows. For iterations $i=1, \ldots, I$

$$
\gamma_{k, s}^{(i)}=\int \sum_{a_{k}} v_{k} \eta_{k, s} \rho_{s}^{(i-1)} \mathrm{d} \boldsymbol{x}_{s},
$$

where the $\gamma_{k, s}$ message contains the measurement corrected information about the state of feature $k$, with the $\mathrm{V} 2 \mathrm{~F}$ measurements from vehicle $s$, where measurement correction has been weighted according to the output of the DA. With the updated $\gamma_{k, s}$ messages, we update $\tilde{\alpha}_{k, s}$ as

$$
\tilde{\alpha}_{k, s}^{(i)}=\alpha_{k} \prod_{s^{\prime} \neq s} \gamma_{k, s^{\prime}}^{(i)} .
$$

We proceed to update $\mu_{k, s}$ as

$$
\mu_{k, s}^{(i)}=\int \sum_{a_{k}} v_{k} \eta_{k, s} \tilde{\alpha}_{k, s}^{(i)} \mathrm{d} \boldsymbol{f}_{k},
$$

and then $\rho_{k, s}$ as

$$
\rho_{k, s}^{(i)}=\phi_{s} \chi_{s} \prod_{k^{\prime} \neq k} \mu_{k^{\prime}, s}^{(i)} .
$$

Step 6 - Computation of posterior beliefs: When I iterations of measurement correction are completed, we fuse the information from all vehicles to form the posterior belief of the state of feature $k$, and update the belief of the vehicle states with the information from feature tracking. The posterior beliefs of the feature states are computed by

$$
b_{k, t}^{(\boldsymbol{f})} \propto \alpha_{k, t} \prod_{s=1}^{S} \gamma_{k, s, t}^{(I)} .
$$

Finally, the vehicle state posterior is computed by

$$
b_{s, t}^{(\boldsymbol{x})}=\phi_{s, t} \chi_{s, t} \prod_{k=1}^{K} \mu_{k, s, t}^{(I)} .
$$

\section{B. Particle-based Representation}

With the possibly nonlinear vehicle and feature state dynamic models, the possible nonlinear measurement models and the DA description, a closed-form expression of (9) is intractable. For this reason, we represent messages and beliefs of continuous random variables (such as $\boldsymbol{x}_{s, t}$ ) by random measures each consisting of a set of $N_{p}$ support points (particles) with associated weights, e.g., $\left\{\boldsymbol{x}_{s, t}^{(l)}, w_{s, t}^{(l)}\right\}_{l=1}^{N_{p}}$, such that the vehicle state belief is expressed as [16]

$$
b_{s, t}^{(\boldsymbol{x})}\left(\boldsymbol{x}_{s, t}\right) \approx \sum_{l=1}^{N_{p}} w_{s, t}^{(l)} \delta\left(\boldsymbol{x}_{s, t}-\boldsymbol{x}_{s, t}^{(l)}\right)
$$

where the weights are normalized such that $\sum_{l=1}^{N_{p}} w_{s, t}^{(l)}=1$, and $\delta$ denotes the Dirac delta distribution (not to be confused with the message $\delta_{m, k}$ ). The particle-based implementation of the message passing algorithm follows the same steps as outlined earlier in the section, with an additional resampling step to avoid particle degeneracy [16]. Products of messages represented by a common list of particles are done by pointwise multiplication of the weights.

\section{Practical Considerations}

We observe that (22) and (25) requires fusing of information from all $S$ vehicles. As each vehicle sends raw measurements (V2F and GNSS) to the RSU, it can perform these operations. In terms of complexity, the proposed algorithm depends on the number of DA iterations $P$, the number of measurement update iterations $I$, the number of vehicles $S$, the number of 


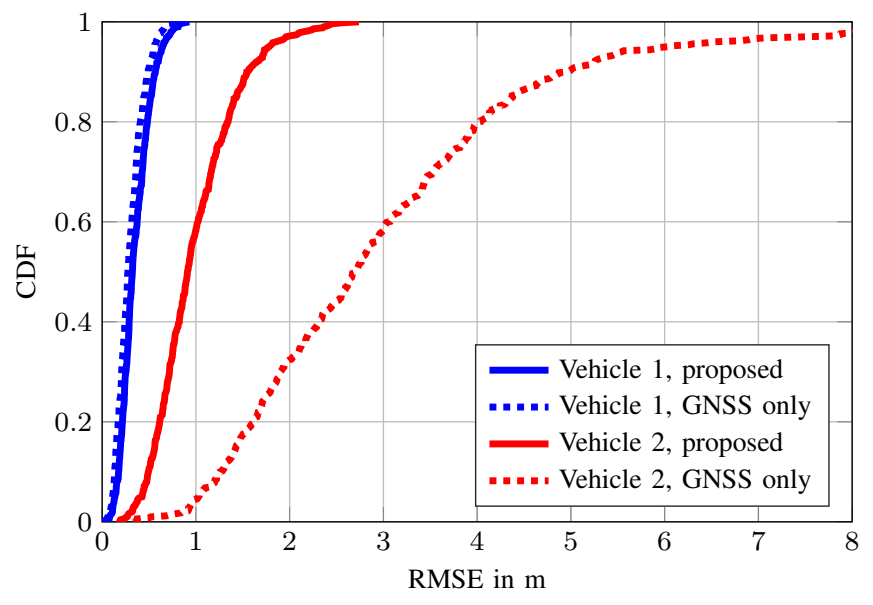

Figure 3. The CDF of the RMSE of the vehicle state estimates is shown for GNSS-only positioning and the proposed method.

features $K$, and the number of particles used to represent a feature/vehicle state $N_{p}$. It scales as $\mathcal{O}\left(\left(K N_{p}\right)^{2} I S P\right)$. For the case the vehicle states are known, complexity scales as $\mathcal{O}\left(K^{2} N_{p} I S P\right)$ as has been shown in [6].

\section{NumERICAL RESUlts AND Discussion}

In this section, we describe first the simulation setup followed by the simulation results and discussion thereof.

\section{A. Setup and Parameters}

We consider a cooperative ITS scenario where the tracking algorithm is run centrally at an RSU involving $S=2$ vehicles, as illustrated in Fig. 1. The state of vehicle $s$ at time instant $t$ is $\boldsymbol{x}_{s, t}=\left[\boldsymbol{p}_{s, t}^{\top}, \boldsymbol{v}_{s, t}^{\top}\right]^{\top}$ with position $\boldsymbol{p}_{s, t} \in \mathbb{R}^{2}$ and velocity $\boldsymbol{v}_{s, t} \in \mathbb{R}^{2}$. Vehicle dynamics follow a constant velocity (CV) model with $\boldsymbol{x}_{s, t}=\boldsymbol{A} \boldsymbol{x}_{s, t-1}+\boldsymbol{r}_{s, t}$. Here,

$$
\boldsymbol{A}=\left[\begin{array}{cc}
1 & \Delta T \\
0 & 1
\end{array}\right] \otimes \boldsymbol{I}_{2},
$$

where $\Delta T=0.5 \mathrm{~s}$, and $\boldsymbol{r}_{s, t} \sim \mathcal{N}(0, \boldsymbol{R})$ with

$$
\boldsymbol{R}=r\left[\begin{array}{cc}
(\Delta T)^{3} / 3 & (\Delta T)^{2} / 2 \\
(\Delta T)^{2} / 2 & \Delta T
\end{array}\right] \otimes \boldsymbol{I}_{2},
$$

where $r=0.1 \mathrm{~m}^{2}$ and the operator $\otimes$ denotes the Kronecker product. The vehicle state is observed through $\boldsymbol{z}_{s, t}^{(\mathrm{G})}=$ $\boldsymbol{H} \boldsymbol{x}_{s, t}+\boldsymbol{w}_{s, t}^{(\mathrm{G})}$, where

$$
\boldsymbol{H}=\left[\begin{array}{l}
1 \\
0
\end{array}\right]^{\top} \otimes \boldsymbol{I}_{2}
$$

and $\boldsymbol{w}_{s, t}^{(\mathrm{G})} \sim \mathcal{N}\left(0, \boldsymbol{W}_{s, t}\right)$ with $\boldsymbol{W}_{s, t}=\sigma_{s}^{(\mathrm{G}), 2} \boldsymbol{I}_{2}$. For vehicle $s=1$, we assume it has low location uncertainty with $\sigma_{s}^{(\mathrm{G}), 2}=10^{-2} \mathrm{~m}^{2}$ and for vehicle $s=2$ high location uncertainty with $\sigma_{s}^{(\mathrm{G}), 2}=15 \mathrm{~m}^{2}$. There are $K=3$ features present, where feature state $\boldsymbol{f}_{k, t} \in \mathbb{R}^{4}$ is comprised of position and velocity, similar to vehicle state $\boldsymbol{x}_{t, s}$. Furthermore, feature dynamics follow the $\mathrm{CV}$ model with the same parameters used for the vehicles. To generate a challenging scenario for DA, we initialize the feature states $\boldsymbol{f}_{k, t} \sim \mathcal{N}\left(0,0.25 \boldsymbol{I}_{4}\right)$ for $t=251 \mathrm{~s}$

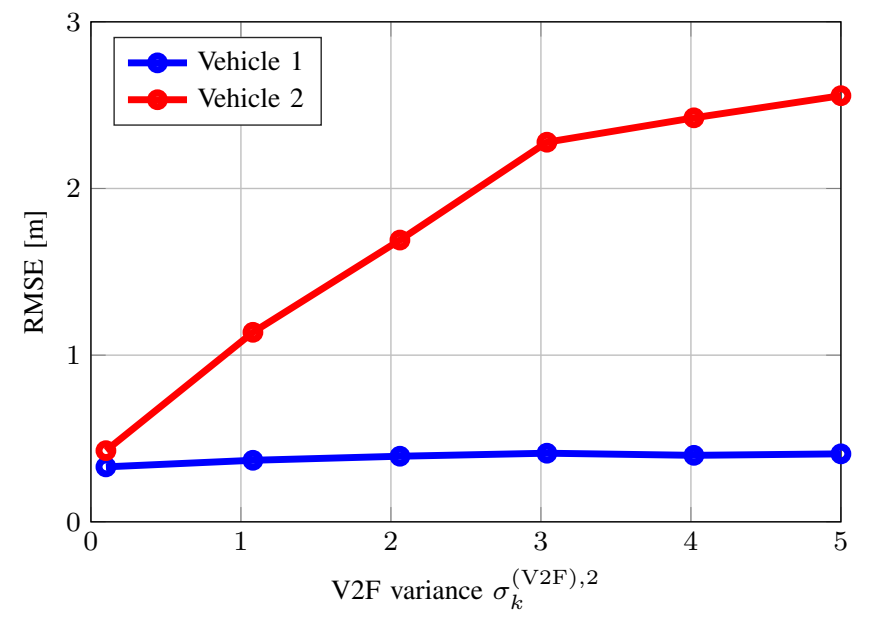

Figure 4. The RMSE averaged over the whole vehicle trajectory is shown for different values of V2F measurement variance $\sigma_{k}^{(\mathrm{V} 2 \mathrm{~F}), 2}$.

and $k=1, \ldots, K$ and run the $\mathrm{CV}$ model forward and backward in time similar to [17, Sec. VI]. The V2F measurement is modeled as $\boldsymbol{z}_{k, s, t}=\boldsymbol{H}^{(\mathrm{V} 2 \mathrm{~F})}\left[\boldsymbol{f}_{k, t}^{\top}, \boldsymbol{x}_{s, t}^{\top}\right]^{\top}+\boldsymbol{w}_{k, s, t}^{(\mathrm{V} 2 \mathrm{~F})}$. Here, $\boldsymbol{H}^{(\mathrm{V} 2 \mathrm{~F})}=[\boldsymbol{H},-\boldsymbol{H}]$ and $\boldsymbol{w}_{k, s, t}^{(\mathrm{V} 2 \mathrm{~F})} \sim \mathcal{N}\left(0, \boldsymbol{W}_{k, s, t}\right)$ with $\boldsymbol{W}_{k, s, t}=\sigma_{k}^{(\mathrm{V} 2 \mathrm{~F}), 2} \boldsymbol{I}_{2}$, where $\sigma_{k}^{(\mathrm{V} 2 \mathrm{~F}), 2}=1 \mathrm{~m}^{2}$ unless stated otherwise. We use $P=5 \mathrm{DA}$ iterations, $I=1$ measurement correction iterations, $N_{p}=600$ particles to represent each state, and we simulate for 501 time-steps.

\section{B. Numerical Results and Discussion}

In Fig. 3, we plot the cumulative distribution function (CDF) for the root mean squared error (RMSE) of the vehicle state using the proposed algorithm. For comparison, we also show the performance of a GNSS-only noncooperative particle filter, i.e., where only GNSS measurements $\boldsymbol{z}_{s, t}^{(\mathrm{G})}$ are considered. Note that, due to the linear observation and motion models used in this simulation, this case corresponds to a Kalman filter tracking the vehicle state. For the GNSS-only case, we observe that vehicle 1 has a low RMSE thanks to the accurate GNSS measurements. In contrast to this, vehicle 2 lacks accurate GNSS measurements leading to a high RMSE. Executing the proposed algorithm, which incorporates the V2F measurements, reduces the RMSE significantly. Similar performance was observed for a multi-vehicle scenario (not shown here) indicating benefits for all vehicles with poor GNSS. In Fig. 4, the RMSE averaged over the whole vehicle trajectory is shown for different values of V2F measurement variance $\sigma_{k}^{(\mathrm{V} 2 \mathrm{~F}), 2}$. For vehicle 1 the RMSE stays almost constant and independent of $\sigma_{k}^{(\mathrm{V} 2 \mathrm{~F}), 2}$. The slight increase of RMSE for increasing $\sigma_{k}^{(\mathrm{V} 2 \mathrm{~F}), 2}$ may be due to the loopy BP processing, where information is not incorporated optimally as would be in a centralized Kalman filter over the joint total state. For vehicle 2 the RMSE increases significantly with increasing $\mathrm{V} 2 \mathrm{~F}$ variance until $\sigma_{k}^{(\mathrm{V} 2 \mathrm{~F}), 2} \approx 3 \mathrm{~m}^{2}$. For even higher values of $\sigma_{k}^{(\mathrm{V} 2 \mathrm{~F}), 2}$ the RMSE increase reduces since the vehicle state is still corrected by the GNSS measurement. In comparison, the RMSE for the GNSS-only noncooperative 


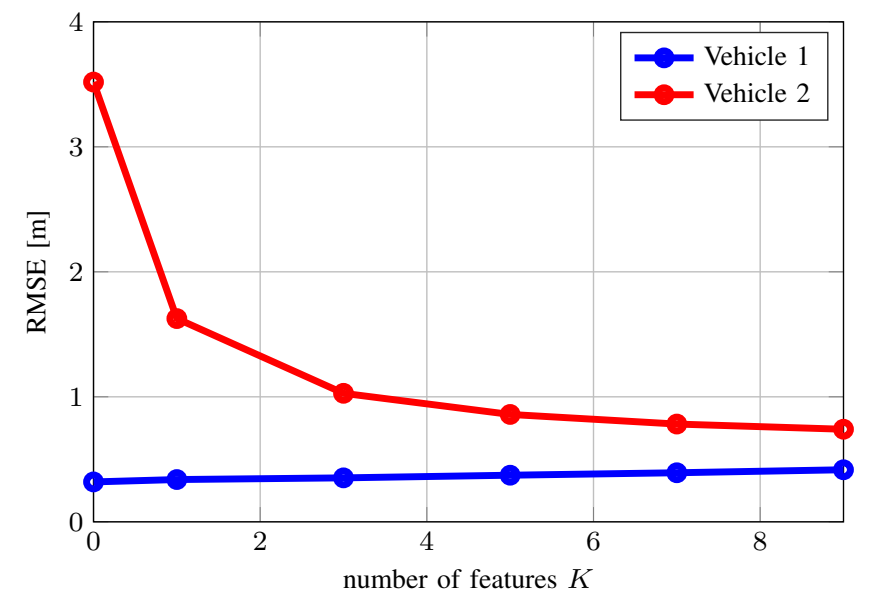

Figure 5. Average RMSE for different number of tracked features. The case with zero features corresponds to independent Kalman filters using GNSS measurements only, which are run separately for vehicle.

Kalman filter is $3.5 \mathrm{~m}$, which can also be seen as the case of feature tracking with $\mathrm{V} 2 \mathrm{~F}$ variance $\sigma_{2}^{(\mathrm{V} 2 \mathrm{~F}), 2}$ approaching infinity. Thus, we can conclude that the accuracy of the V2F measurements plays a significant role about how much information can be transferred from a well-localized vehicle (vehicle 1) to vehicles with a lower accuracy (vehicle 2). In Fig. 5, the RMSE averaged over the whole vehicle trajectory is shown for different number of tracked features. The case where the number of tracked features equals zero corresponds to the GNSS-only noncooperative Kalman filter, executed separately for each vehicle. From the figure, we observe that for vehicle 1 the average RMSE remains almost constant since the GNSS measurement of vehicle 2 is not very informative. For vehicle 1 , no improvement is seen with increasing number of tracked features $K$. As mentioned earlier, the slight increase of RMSE for vehicle 1 for increasing $K$ may be caused by the loopy BP processing. In contrast, vehicle 2 benefits when features are tracked from both vehicles. Furthermore, the RMSE of vehicle 2 decreases with increasing number of tracked features $K$. In this way, accurate feature state information from vehicle 1 allows to reduce the average RMSE of vehicle 2. Increasing the number of tracked features can further reduce the average vehicle state RMSE.

\section{CONCLUSION}

A method for implicit cooperative vehicle state estimation via feature tracking was proposed. Since with vehicle-tofeature measurements the data association between features and measurements is in general not known, it was considered in a suitable factor graph representation. Running nonparametric belief propagation on a road-side unit enables accurate tracking of the vehicle states through cooperative feature tracking. The proposed method scales linearly with the number of vehicles, and quadratically with the number of features. Numerical results demonstrated the improved tracking performance compared to a noncooperative Kalman filter. Future work will consider the incorporation of a time varying number of features as well as non-overlapping sensor fields of view.

\section{ACKNOWLEDGMENT}

This work is supported, in part, by the EU-H2020 project HIGHTS (High Precision Positioning for Cooperative ITS Applications) under grant no. MG-3.5a-2014-636537 and COPPLAR (campus shuttle cooperative perception and planning platform) project, funded under grant no. 2015-04849 from Vinnova.

\section{REFERENCES}

[1] H. Wymeersch, G. R. de Campos, P. Falcone, L. Svensson, and E. G. Ström, "Challenges for cooperative ITS: Improving road safety through the integration of wireless communications, control, and positioning," in Proceedings of the International Conference on Computing, Networking and Communications, pp. 573-578, Mar. 2015.

[2] F. Meyer, O. Hlinka, H. Wymeersch, E. Riegler, and F. Hlawatsch, "Distributed localization and tracking of mobile networks including noncooperative objects," IEEE Transactions on Signal and Information Processing over Networks, vol. 2, pp. 57-71, Mar. 2016.

[3] R. Olfati-Saber, J. A. Fax, and R. M. Murray, "Consensus and Cooperation in Networked Multi-Agent Systems," Proceedings of the IEEE, vol. 95, pp. 215-233, Mar. 2007.

[4] G. Soatti, M. Nicoli, N. Garcia, B. Denis, R. Raulefs, and H. Wymeersch, "Implicit Cooperative Positioning in Vehicular Networks," IEEE Trans. on Intelligent Transportation Systems (to appear), 2018.

[5] F. Meyer, P. Braca, P. Willett, and F. Hlawatsch, "Scalable Multitarget Tracking Using Multiple Sensors: A Belief Propagation Approach," in Proceedings ot the 18th International Conference on Information Fusion, pp. 1778-1785, Sep. 2015.

[6] F. Meyer, P. Braca, P. Willett, and F. Hlawatsch, "A scalable algorithm for tracking an unknown number of targets using multiple sensors," IEEE Transactions on Signal Processing, vol. 65, pp. 3478-3493, Jul. 2017.

[7] J. Williams and R. Lau, "Approximate Evaluation of Marginal Association Probabilities with Belief Propagation," IEEE Transactions on Aerospace and Electronic Systems, vol. 50, pp. 2942-2959, Oct. 2014.

[8] R. L. Streit and T. E. Luginbuhl, "Probabilistic Multi-Hypothesis Tracking," tech. rep., Naval Underwater Systems Center Newport RI, 1995.

[9] R. P. Mahler, Advances in Statistical Multisource-Multitarget Information Fusion. Artech House, 2014.

[10] B.-N. Vo and W.-K. Ma, "The Gaussian Mixture Probability Hypothesis Density Filter," IEEE Transactions on Signal Processing, vol. 54, pp. 4091-4104, Nov. 2006

[11] G. M. Hoang, B. Denis, J. Härri, and D. T. M. Slock, "On communication aspects of particle-based cooperative positioning in GPS-aided VANETs," in Proceedings of the IEEE Intelligent Vehicles Symposium, pp. 20-25, Jun. 2016.

[12] R. P. Mahler, Statistical multisource-multitarget information fusion. Artech House, Inc., 2007.

[13] A. T. Ihler, W. F. John III, and A. S. Willsky, "Loopy Belief Propagation: Convergence and Effects of Message Errors," Journal of Machine Learning Research, vol. 6, pp. 905-936, May 2005.

[14] H. Wymeersch, J. Lien, and M. Z. Win, "Cooperative Localization in Wireless Networks," Proceedings of the IEEE, vol. 97, pp. 427-450, Feb. 2009.

[15] J. L. Williams and R. A. Lau, "Convergence of loopy belief propagation for data association," in Proceedings of the IEEE 6th International Conference on Intelligent Sensors, Sensor Networks and Information Processing, pp. 175-180, Feb. 2010.

[16] M. S. Arulampalam, S. Maskell, N. Gordon, and T. Clapp, "A Tutorial on Particle Filters for Online Nonlinear/Non-Gaussian Bayesian Tracking," IEEE Transactions on Signal Processing, vol. 50, pp. 174-188, Feb. 2002.

[17] J. L. Williams, "Marginal multi-bernoulli filters: RFS derivation of MHT, JIPDA, and association-based MeMBer," IEEE Transactions on Aerospace and Electronic Systems, vol. 51, pp. 1664-1687, Jul. 2015. 